\title{
Adapting a Geriatrics Health Counseling Virtual Agent for the Chinese Culture
}

\author{
Zhe Zhang, ${ }^{1}$ Ha Trinh, ${ }^{1}$ Qiong Chen, ${ }^{2}$ Timothy Bickmore ${ }^{1}$ \\ ${ }^{1}$ College of Computer and Information Science, Northeastern University, Boston, MA \\ ${ }^{2}$ Xin Hua Hospital, Shanghai, China \\ \{zessiez,hatrinh,bickmore\}@ccs.neu.edu
}

\begin{abstract}
The design of a virtual conversational agent that provides cardiovascular health counseling to hospitalized geriatrics patients in China is described, along with the linguistic and cultural adaptations performed to tailor the agent for China. Results of a preliminary study comparing conversations with the agent to conversations with a geriatrician in a hospital in Shanghai demonstrated high levels of patient acceptance and satisfaction with the agent, although not as high as for the human doctor.
\end{abstract}

\section{Introduction}

With its aging population, mandatory retirement, and one-child policy, China is facing a crisis in its ability to care for its elderly. There will not be nearly enough caregivers to meet the demand of individuals who want to live independently as long as possible. Virtual agents provide a solution to automating many routine healthcare tasks, and agents that play the role of health counselors have been shown to be wellaccepted by older adults in several cultures [1]. To help address these issues, we have developed a virtual agent to counsel geriatrics patients about cardiovascular health (Figure 1). Cardiovascular conditions, such as stroke and myocardial infarction are among the leading causes of morbidity and mortality for Chinese older adults. Standard prevention measures include appropriate exercise, diet, and stress management, and adherence to prescribed medications.

Culture is important in health education interventions. Many studies have demonstrated the importance of cultural congruity between health providers and their patients. For example, patients usually prefer health counselors from their own culture due to their presumed familiarity with cultural values [2].

Our general approach was to first develop the agent for the English-speaking Anglo-American culture we are most familiar with, then methodically adapt it, linguistically and culturally, for the Chinese older adult population. The agent counsels patients on their diagnoses and medications specified by a clinician, as well as increasing physical activity, improving diet, decreasing stress, and motivating them to be more involved and proactive in their own care. The virtual agent speaks using synthetic speech driven by a dialogue engine, and has a range of nonverbal behaviors that it can use, including facial displays of emotion, hand gestures, body posture 


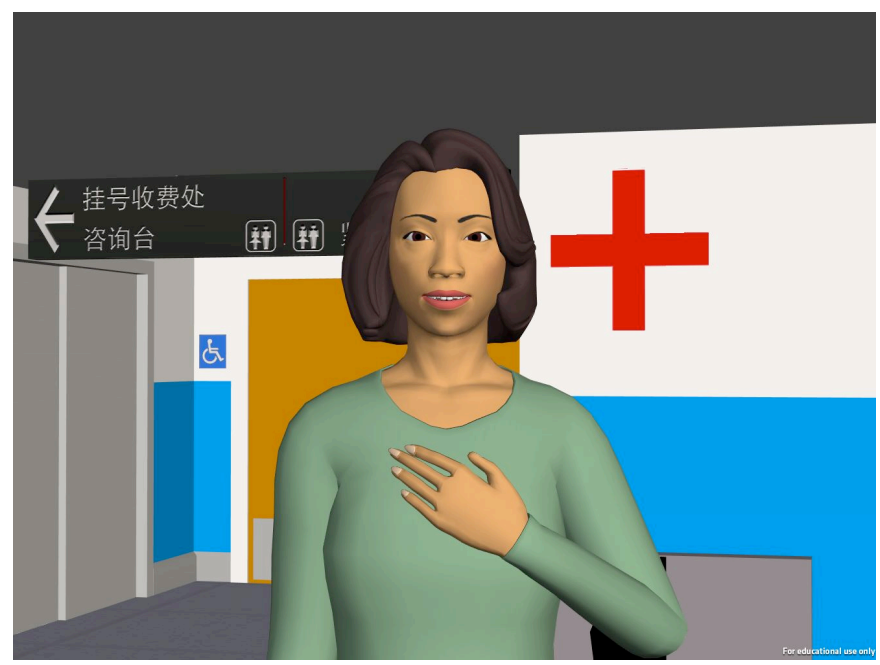

Fig. 1. Chinese Geriatrics Cardiovascular Health Counselor

shifts, gaze shifts, eyebrow raises, and head nods. The entire counseling system was deployed on an 8 " touchscreen tablet to facilitate use in a hospital environment.

\section{Adaptation for the Chinese Culture}

We began our cultural adaptation of the agent following Hofstede's framework [3]. Two dimensions stand out as particularly important for differentiating US and Chinese cultures: power-distance and individualism-collectivism. Physicians are at the top of the hierarchy in the US healthcare system and are highly regarded in healthcare and society in general. Thus, we initially assumed that in a high powerdistance culture such as China, a virtual doctor should appear as a socially remote, authoritative figure. However, after our discussions with Chinese doctors and patients, we found that the situation in China is different. Healthcare in China is not entirely subsidized by the government, and the population has an extreme consumerist attitude about the healthcare system, demanding service and outcomes for their money. Given this, we designed our virtual health counselor to appear as more of an approachable and friendly peer, without the official trappings of a lab coat and stethoscope.

Given the greater bias towards collectivism in China compared to the US, we did weave elements of collectivism into the translated counseling scripts. For instance, we designed a particular coaching strategy to encourage patients to work together with their family members and close friends on their health conditions. Also, when motivating patients to adhere to different aspects of their medical regimen, we emphasized how their activity could potentially affect their family members.

Virtual Counselor Appearance. Prior research has highlighted the importance of the agent appearance for perceptions of cultural congruity. Accordingly, we tailored the 
virtual counselor character model to have more of a Chinese appearance, adjusting its skin-tone to be more yellowish and tailored its hair to be dark brown. We also designed black irises for the character to match Asian genetics. We designed a background for the character to match images of Chinese hospital interiors (Figure 1).

Dialogue. Conversational dialogue is the primary communication avenue for the virtual agent to interact with its users. The dialogue content mainly focuses on topics such as cardiovascular diseases, associated medications, and physical activity, diet, and stress management, all considered common topics of physician-patients conversations in China. These dialogues are designed and delivered based on each patient's particular health conditions and doctors' recommendations. To make the dialogues culturally appropriate to Chinese patients, contextual adaptation was applied. For instance, we adapted "How are you doing today?" to a linguistically equal version of "How are you feeling today?" in Chinese based on how a doctor typically initiates a conversation with a patient in China. Also, we removed the "serving" concept from the fruit and vegetable promotion part of the diet dialogue given that it is not commonly used in Chinese culture, and adopted "bowl" to quantitatively measure vegetable and fruit intake. Finally, in the farewell dialogue, we replaced "Have a nice day" with "I wish you good health", which is the most common way to end a medical conversation in China.

Nonverbal Behavior. Our system automatically generates a number of nonverbal behaviors for the agent using our extended version of the Behavior Expression Animation Toolkit (BEAT) [4]. BEAT assigns nonverbal behaviors based on linguistic and contextual analysis of the agent's speaking text. As BEAT was originally developed for English, we extended its language module to perform syntactic and discourse analysis for the Chinese language. Our system annotates the Chinese text with grammatical structure information using the Stanford part-of-speech tagger and a factored parser trained on Xinhua newspaper text from Mainland China.

\section{Pilot Acceptance Study}

We collaborated with clinicians from Xin Hua Hospital in China, on a preliminary, between-subjects study to evaluate the acceptance and usability of the agent system by local geriatrics patients. Our aim was to assess the patients' overall satisfaction with the system and their attitude towards the culturally tailored agent following a brief counseling session, in comparison with a human geriatrician.

Participants. We recruited a total of 10 patients: $80 \%$ male, aged from 70 to 94 (mean $=85.89$ ), $80 \%$ were inpatients, $60 \%$ had high school or higher degrees, $50 \%$ had high level of computer literacy, 50\% reported high level of confidence in filling out the forms by themselves. The patients had a variety of health conditions and occupational backgrounds, including professor, retired civil servant, military veteran, office clerk, and doctor. Six of them were randomized into the intervention group. 
Measures. The acceptance and use of the system was evaluated using the 7-point scales outlined in Table 1.

Table 1. Patients' Ratings of The Culturally Adapted Agent System

\begin{tabular}{|l|l|l|}
\hline \multicolumn{1}{|c|}{ Rating Aspects (Scale Measures from 1-7) } & $\begin{array}{c}\text { Agent } \\
\text { Mean (SD) }\end{array}$ & $\begin{array}{c}\text { Physician } \\
\text { Mean (SD) }\end{array}$ \\
\hline How satisfied were you? (1-not at all, 7-very satisfied) & $4.67(1.75)$ & $6.00(0.00)$ \\
\hline $\begin{array}{l}\text { How much would like to continue working with the } \\
\text { counselor/doctor? (1-not at all, 7-very much) }\end{array}$ & $4.83(1.60)$ & $6.50(0.58)$ \\
\hline $\begin{array}{l}\text { How much do you trust the counselor/doctor? (1-not at all, 7- } \\
\text { very much) }\end{array}$ & $5.33(1.21)$ & $6.75(0.50)$ \\
\hline $\begin{array}{l}\text { How much do you like the counselor/doctor? (1-not at all, 7- } \\
\text { very much) }\end{array}$ & $4.83(1.60)$ & $6.75(0.50)$ \\
\hline $\begin{array}{l}\text { How easy was talking to the counselor/doctor? (1-easy, 7- } \\
\text { difficult) }\end{array}$ & $2.17(1.94)$ & $1.25(0.50)$ \\
\hline $\begin{array}{l}\text { How would you characterize your relationship with the } \\
\text { counselor/doctor? (1-complete stranger, 7-close friend) }\end{array}$ & $3.67(1.63)$ & $5.25(0.50)$ \\
\hline $\begin{array}{l}\text { How much do you feel the counselor/doctor cares about you? } \\
\text { (1-not at all, 7-very much) }\end{array}$ & $3.83(1.47)$ & $7.00(0.00)$ \\
\hline $\begin{array}{l}\text { How likely is it that you will follow the advice? (1-not at all, } \\
\text { 7-very likely) }\end{array}$ & $6.00(1.55)$ & $7.00(0.00)$ \\
\hline
\end{tabular}

Quantitative Results. Although not as high as for the human doctor, patients were highly satisfied with the virtual counselor, exhibited high desire to continue working with her, trusted and liked her, and would follow her advices in the future (Table 1). In addition, patients who had high school or higher degrees expressed significantly more trust in the virtual counselor than those whose degrees are lower than high school $(\mathrm{p}<.05)$. Patients with high computer literacy felt that the agent cared more about them, compared to those with low computer literacy $(\mathrm{p}<.05)$.

Conclusion. Patients in our study responded very well to the agent, expressing high levels of trust, engagement and respect for the agent. Future work includes extending the counseling content with more culturally appropriate health topics, while improving the visual appearance and nonverbal behavior of the agent and the quality of the agent's speech.

Acknowledgments. Thanks to Shuo Zhou and Lin Shi for review and input, and Zachary Berwaldt for work on the character model.

\section{References}

1. King, A., et al., Employing 'Virtual Advisors' in Preventive Care for Underserved Communities: Results from the COMPASS Study. Journal of Health Communication, 2013. 18(12): p. 1449-1464.

2. Sue, D., A. Ivey, and P. Pederson, Theory of Multicultural Counseling \& Therapy. 2007: Thomson Brooks/Cole.

3. Hofstede, G., Culture's Consequences. 2003: Sage.

4. Cassell, J., H. Vilhjálmsson, and T. Bickmore. BEAT: The Behavior Expression Animation Toolkit. in SIGGRAPH '01.2001. Los Angeles, CA. 